\title{
Unexpected death secondary to streptococcus salivarius endocarditis
}

\author{
Butch M Huston*, Victor Froloff, Kelly Mills, Michael McGee \\ Ramsey County Medical Examiner's Office, Minnesota, United States
}

Received: September 4, 2019

Accepted: October 8, 2019

Online Published: October 19, 2019

DOI: $10.5430 /$ crep.v6n1p23

URL: https://doi.org/10.5430/crcp.v6n1p23

\begin{abstract}
A 51-year old male with a medical history of chronic ethanol and tobacco use was complaining of difficulty breathing and was driven to the local emergency department where he was noted to be in respiratory distress. The decedent had not seen a physician in over ten years. The decedent was undergoing an ultrasound to evaluate for a lower extremity deep venous thrombosis when he arrested. He was unable to be resuscitated. An autopsy was performed. The external examination revealed numerous petechial type hemorrhages of the skin with prominence of the arms and lower legs. Internal examination revealed cardiomegaly (heart weight 720 grams) with left ventricular hypertrophy and prominent endocarditis of the mitral and aortic valves. Infarcts were noted involving the right kidney and the spleen. A postmortem blood culture grew Streptococcus salivarius in both the aerobic and anaerobic culture bottles.
\end{abstract}

Key Words: Streptococcus salivarius, Endocarditis, Forensic pathology, Sudden death

\section{Case presentation}

The decedent was a 51-year old male with a medical history of chronic ethanol and tobacco use. According to family, the decedent had not used alcohol in several years. The decedent had not seen a physician in over ten years. On the date of death, the decedent complained of difficulty breathing and was driven to the local emergency department by a family member where he was noted to be in respiratory distress. Suspecting a possible pulmonary embolus, the decedent was sent for an ultrasound of the lower legs to evaluate for a deep venous thrombosis. The decedent arrested during the ultra sound procedure. He was unable to be resuscitated. An autopsy was performed. The external examination revealed numerous petechial type hemorrhages of the skin, particularly noted involving the arms and lower legs (see Figure 1). The decedent's dentition appeared grossly unremarkable and the decedent had not had any recent dental procedures according to family.

An examination of the heart revealed a smooth and glistening pericardial surface. There was a small amount of strawcolored fluid within the pericardial sac. The coronary arteries showed a normal distribution. No significant atherosclerosis was identified. The myocardium was red-brown with no masses or defects. The cardiac valves were in their usual anatomic positions. The tricuspid and pulmonary valve appeared unremarkable. The mitral valve displayed a large area of vegetation above the anterior cusp measuring approximately $2 \mathrm{~cm}$ in greatest dimension that partially occluded the lumen of the valve (see Figure 2). The aortic cusps were not identifiable and were replaced by numerous vegetations occluding the lumen of the valve (see Figure 3 ). There was no significant dilation of the atria or ventricles. The left ventricle measured $1.6 \mathrm{~cm}$ in thickness concentrically. The

\footnotetext{
*Correspondence: Butch M Huston; Email: Butch.huston@ co.ramsey.mn.us; Address: Ramsey County Medical Examiner's Office, 300 E University Ave.,St. Paul, MN, United States.
} 
right ventricle measured $0.3 \mathrm{~cm}$ in thickness.

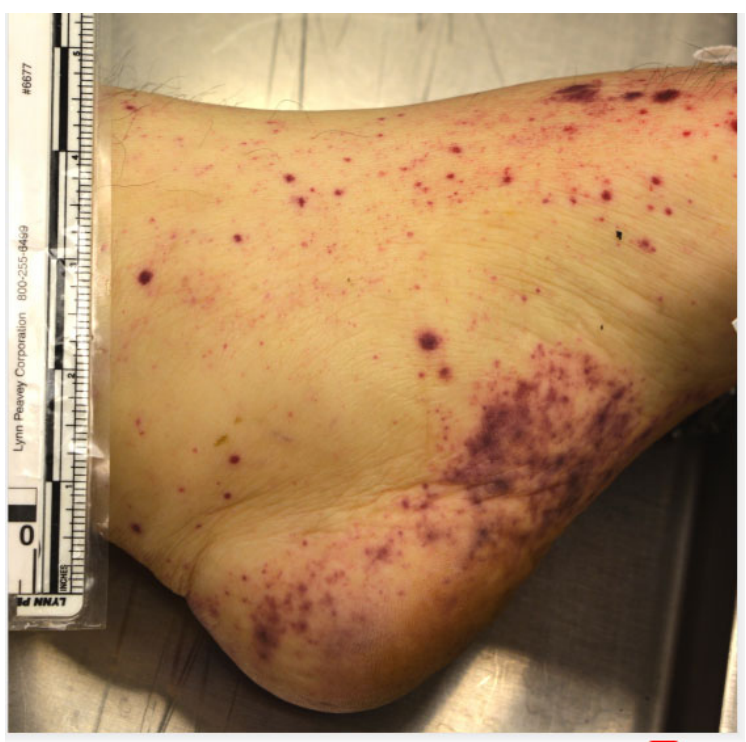

Figure 1. A photograph of the medial aspect of the left foot shows multiple petechial hemorrhages on the skin of the decedent

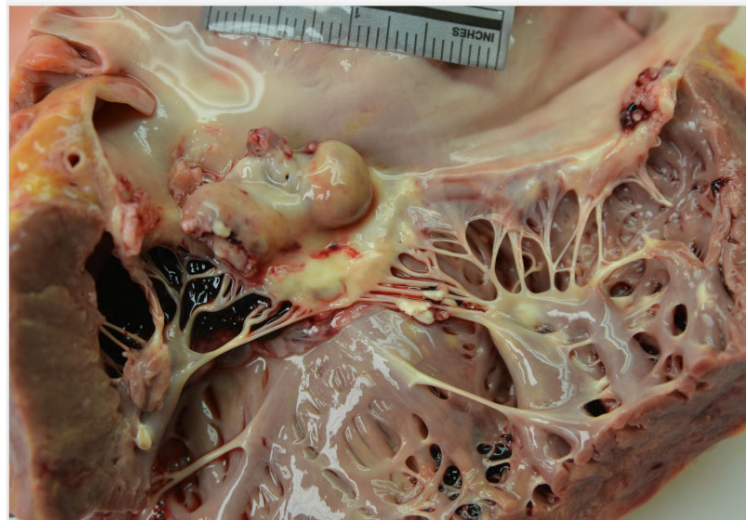

Figure 2. The mitral valve shows a large vegetation which was noted to partially occlude the lumen of the valve

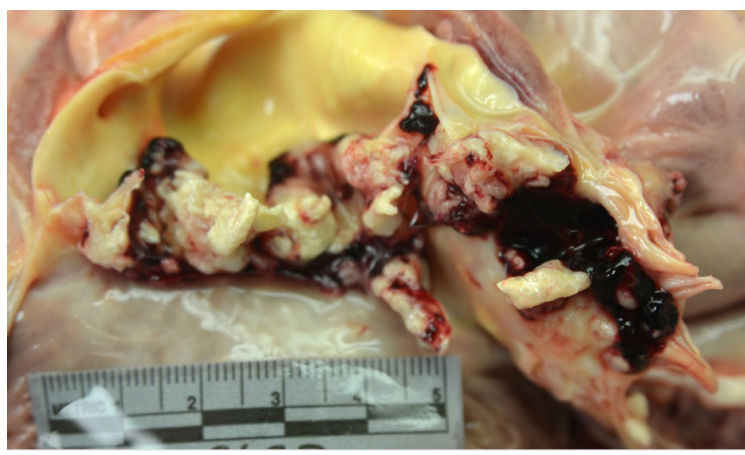

Figure 3. The aortic cusps were noted to be replaced by numerous vegetations occluding the lumen of the valve. There is thrombus noted adherent to the vegetations
Approximately $500 \mathrm{cc}$ of straw-colored fluid was noted in the right and left pleural spaces. Examination of the lungs revealed severe pulmonary edema and congestion the right lung weighing 1,490 grams and the left lung weighing 1,160 grams.

The capsules of the kidneys stripped with ease revealing an underlying red-brown, smooth, cortical surface. The right kidney showed a large area of necrosis measuring $2 \mathrm{~cm}$ in greatest dimension (see Figure 4). The parenchyma was otherwise unremarkable.

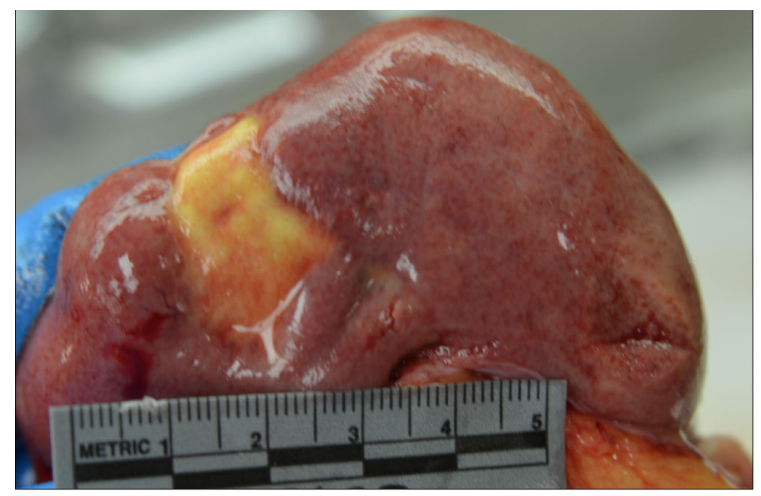

Figure 4. The exterior surface of the right kidney showed a large area of necrosis measuring $2 \mathrm{~cm}$ in greatest dimension

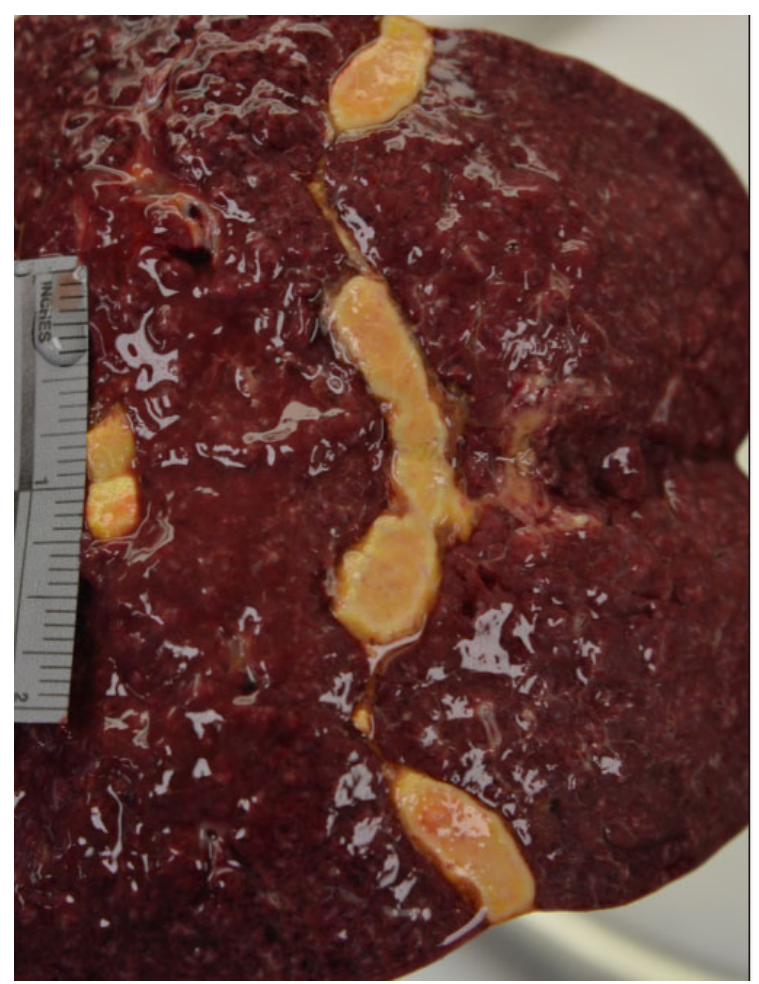

Figure 5. A cross section of the spleen revealed numerous areas of tan-yellow, well demarcated, areas of necrosis ranging in size from $1 \mathrm{~cm}$ to $2 \mathrm{~cm}$ in greatest dimension 
The outer surface of the spleen was smooth and grey-purple. The parenchyma was dark red with numerous areas of tanyellow, well demarcated, areas of necrosis ranging in size from 1 to $2 \mathrm{~cm}$ in greatest dimension (see Figure 5).

Histologic examination of the mitral valve revealed diffuse areas of necrosis, hemorrhage, acute inflammatory infiltrate, fibrosis and focal calcification (see Figure 6). Abundant clusters of bacteria were noted (see Figure 7). A tissue gram stain revealed abundant gram positive cocci in clusters and chains (see Figure 8). Microscopic examination of the cardiac tissue revealed multifocal areas of interstitial and perivascular fibrosis with a mixed inflammatory infiltrate (see Figure 9). The spleen and kidney showed histologic evidence of infarction.

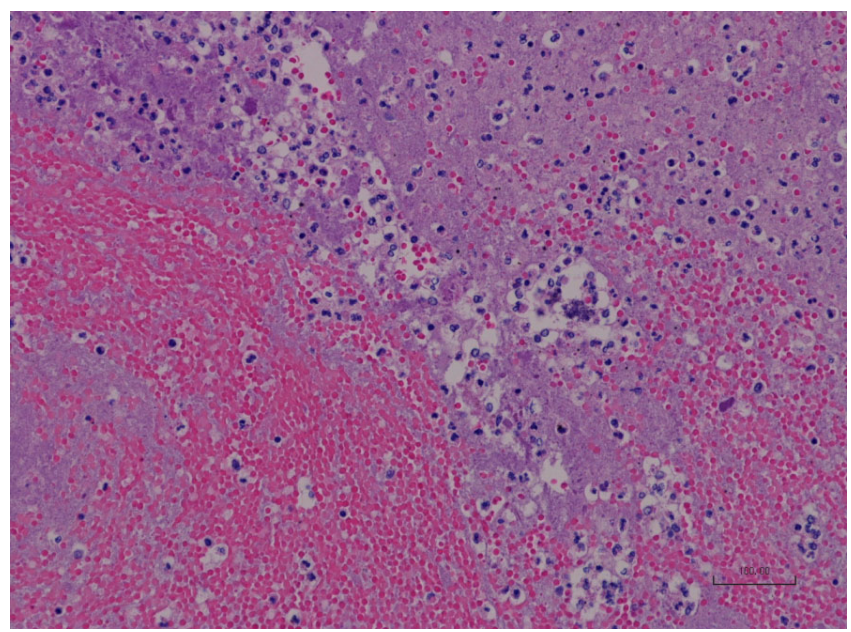

Figure 6. A histologic section of the mitral valve revealed diffuse areas of necrosis, hemorrhage, acute inflammatory infiltrate, fibrosis and focal calcification. (original magnification, 40X)

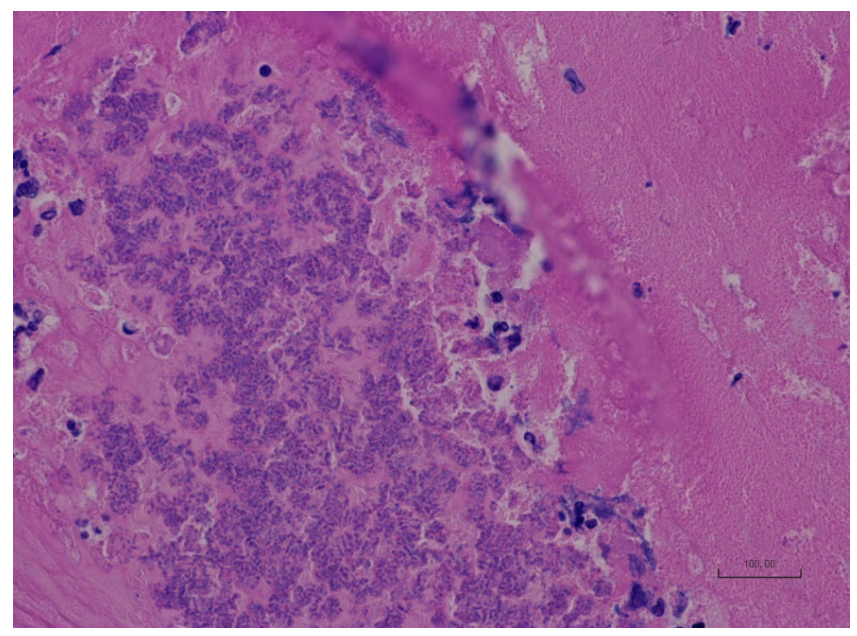

Figure 7. The mitral valve histologic section showed abundant clusters of bacteria. (original magnification, 400X) Published by Sciedu Press

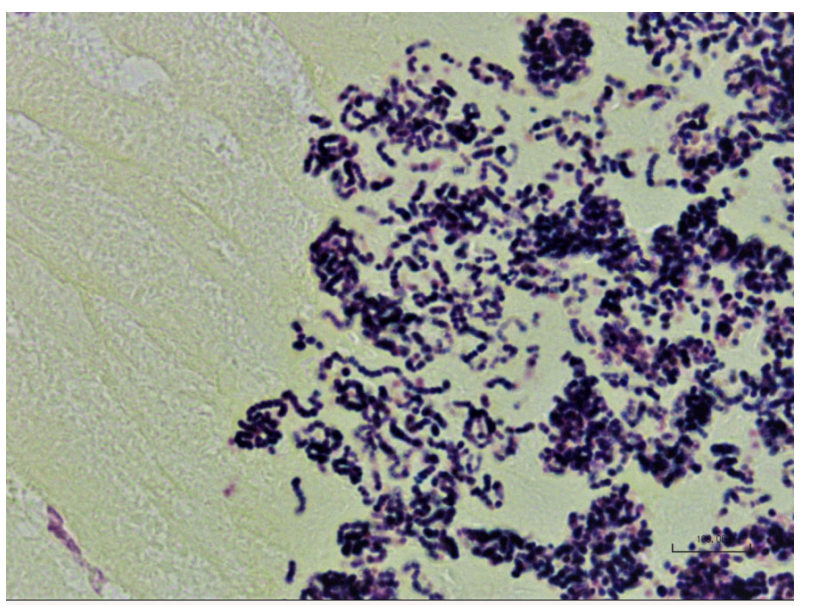

Figure 8. A tissue gram stain of the mitral valve revealed abundant gram positive cocci in clusters and chains. (original magnification, 1000X)

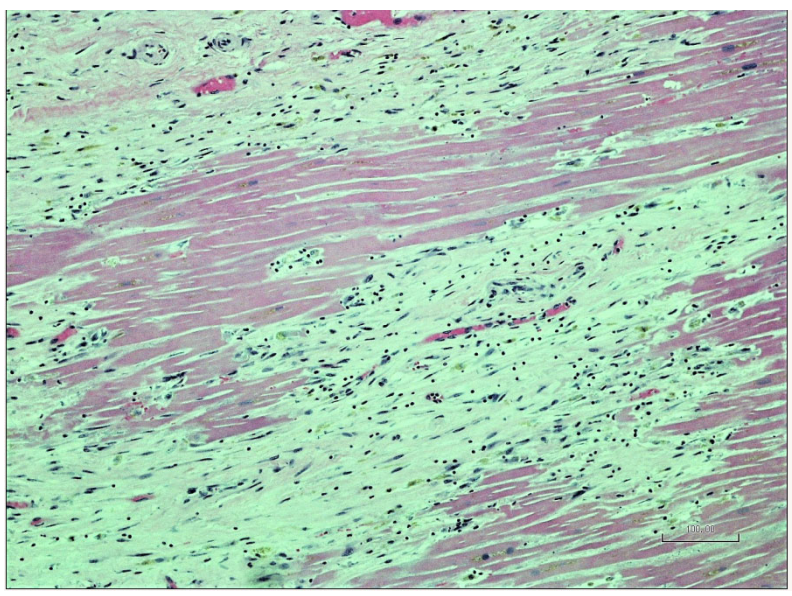

Figure 9. Microscopic examination of the cardiac tissue revealed multifocal areas of interstitial and perivascular fibrosis with a mixed inflammatory infiltrate. (original magnification, 20X)

A postmortem blood culture grew Streptococcus salivarius bacteria in both the aerobic and anaerobic culture bottles.

\section{Discussion}

Streptococcus viridans group are comprised of diverse bacteria that can be divided into salivarius, mitis, anginosus and mutans groups. Each group contains multiple species and subspecies. Streptococcus salivarius group contains the members Streptococcus salivarius and Streptococcus vestibularis. Streptococcus salivarius is a gram positive cocci that is catalase and oxidase negative and is noted in pairs and chains. S. salivarius is a normal colonizer of the oral cavity and upper respiratory tract. The bacteria are considered an opportunistic pathogen, rarely finding its way into the bloodstream, where it has been implicated in cases of sepsis in people with 
neutropenia. Streptococcus species are commonly associated with dental caries and have been reported to result in bacterial endocarditis following dental procedures. There may be increasing cases of infectious endocarditis over the last few decades because cardiac procedures, implanted cardiac devices and indwelling catheters are increasingly utilized. ${ }^{[1]}$

The incidence of infective endocarditis (IE) in the United States is approximately $3-15$ per $100,000^{[1,2]}$ and with Streptococci occurring from 26-42/million annually. ${ }^{[2]}$ Symptoms of IE can include fever, sweats, extremity swelling, myalgia, fatigue and increased heart rate. Purple discoloration of the fingertips, splinter hemorrhages of the fingernails and petechial hemorrhages can also be identified in some cases of bacteremia. ${ }^{[3]}$ IE can be diagnosed with a positive blood culture and supported with a transthoracic echocardiogram. Microbiological diagnosis can be determined by the blood culture, or tissue culture if the valve is surgically removed. Tissue can be used to perform a molecular diagnosis by PCR in order to determine the exact bacterial organism. ${ }^{\text {[] }}$ Determining the causative bacteria in IE is important to determine the appropriate antibiotic treatment. S. salivarius is closely related to $\mathrm{S}$. bovis. Differentiating between these two bacteria is also important since $\mathrm{S}$. bovis is noted to have a greater association with colon cancer. ${ }^{[5,7]}$

Streptococcus salivarius is a rare pathogen that has been reported to cause IE. Often, it can be difficult to determine if a positive blood culture is a contaminant, or clinically relevant. A 16-year prospective study indicated that $32 \%$ of S. salivarius blood cultures were clinically significant. ${ }^{[6]}$ Another report found that this species comprised approximately $1 \%$ of IE cases in their study. ${ }^{[4]}$

Streptococcus salivarius has also been reported to result in bacteremia and meningitis following an upper endoscopy and cauterization for gastric hemorrhage. ${ }^{[8]}$

Another case reports a pontine abscess after initial ineffective treatment for IE. ${ }^{[9]}$ This case illustrates that continued medical follow-up may be necessary even with an initial correct identification of the bacterial endocarditis and proper antibiotic treatment.

\section{CONFlicts OF InTEREST Disclosure}

The authors declare that they have no competing interest.

\section{REFERENCES}

[1] Cahill TJ, Prendergast BD. Infective Endocarditis. Lacet. 2016; 387: 882-893. https://doi.org/10.1016/S0140-6736(15)0 0067-7

[2] Pant S, Patel NJ, Deshmukh A, et al. Trends in Infective Endocarditis Incidence, Microbiology, and Valve Replacement in the United States from 2000-2011. J AM Coll Cardiol. 2015; 65(19): 2070-2076. PMid:25975469. https://doi.org/10.1016/j.jacc.2015.03 .518

[3] Chawla D, Rhee Y, Singh K. Society of General Internal Medicine (SGIM). Available from: https: //www.sgim.org/web-only/clinical-images/endoca rditis-with-confusion-and-hand-pain

[4] Kim SL, Gordon SM, Shrestha NK. Distribution of streptococcal groups causing infective endocarditis: a descriptive study. Diagn Microbiol Infect Dis. 2018 Jul; 91(3): 269-272. PMid:29567126. https://doi.org/10.1016/j.diagmicrobio.2018.02.015

[5] Felix L, Gurunathan R. "I Can't Believe It's Not Bovis": A Case of Streptococcus Salivarius Related Endocarditis [abstract]. Available from: https://www. shmabstracts. com/abstract/i-cant-b elieve-its-not-bovis-a-case-of-streptococcus-saliv arius-related-endocarditis/. Accessed November 5, 2018.

[6] Corredoira JC, Alonso MP, García JF, et al. Clinical characteristics and significance of Streptococcus salivarius bacteremia and Streptococcus bovis bacteremia: a prospective 16-year study. Eur J Clin Microbiol Infect Dis. 2005 Apr; 24(4): 250-5. PMid:15902530. https ://doi.org/10.1007/s10096-005-1314-x

[7] Abdulamir AS, Hafidh RR, Bakar FA. The Association of Streptococcus bovis/galloyicus with colorectal tumors: The nature and underlying mechanisms of its etiological role. J Exper \& Clinical Research. 2011; 30: 11. PMid:21247505. https://doi.org/10.1186/17 56-9966-30-11 http: //www. jeccr. com/content/30/11

[8] Carley NH. Streptococcus Salivarius Bacteremia and Meningitis Following Upper Gastrointestinal Endoscopy and Cauterization for Gastric Bleeding. Clin Infect Dis. 1992; 14(4): 947-948. PMid:1576292. https://doi.org/10.1093/clinids/14.4.947

[9] Knudtzen FC, Lynge M, Gaini S. Pontine abscess with initial treatment failure following infectious endocarditis with Streptococcus salivarius. BMJ Case Rep. 2015 Jul 2; 2015. PMid:26139646. https ://doi.org/10.1136/bcr-2014-205949 